\title{
A Model of the Deviation between the Intended and the Actual Experiences with Interactive Installations
}

\author{
Dingyi Wei \\ The Bartlett School of Architecture, UCL, London, UK, dingyi.wei.16@ucl.ac.uk \\ Ava Fatah gen. Schieck \\ The Bartlett School of Architecture, UCL, London, UK, ava.fatah@ucl.ac.uk \\ Nicolai Marquardt \\ UCL Interaction Centre, UCL, London, UK, n.marquardt@ucl.ac.uk
}

\begin{abstract}
Interactive installations engage people in different ways, many of which are different than originally intended by the designer. We present a model that illustrates the differences between the intended experience and the actual experience of people. The model is demonstrated through the analysis of one interactive installation at the Science Museum (London) and the various parameters are mapped and visualized. We suggest that the participants of interactive installations engage differently than originally intended by the designer, which can be defined here as "deviation". There are several levels of deviation, and the proposed model will illustrate the critical interaction stages and visualize the deviations. This model offers new tools for designers and curators alike.
\end{abstract}

\section{CCS CONCEPTS}

- Human-centered computing ${ }^{\sim}$ Interaction design ${ }^{\sim}$ Interaction design theory, concepts and paradigms

\section{KEYWORDS}

Interactive installations, Engagement, Intended experience, Deviated behaviors

\section{Introduction}

Interactive installations nowadays are able to perform a variety of functionalities and goals. They can be aesthetically appealing, create playfulness, raise certain awareness, contribute to learning, etc. For example, cases show that interactive experiences can help participants learn [ $\underline{3}]$ and playful behaviors can be transformed into the process of discovering information []ㅡ [ $]$ ].

Participants' performance and interactions do not always match the interaction designers' intention and design goals. Early research reported, for instance, the reluctance by the audience [2]. Others observed the unintended behaviors throughout the interaction [1]. Interactive installations involve complex factors that influence the overall interactions and could trigger encounters with many participants whose behaviors are rather difficult to predict. To help understand this gap, we suggest it is necessary to provide a tool that can capture and visualize the difference between the experience anticipated by the designer and the actual experience of the participants on a variety of interaction stages. This difference can be referred to as the "deviation" between the two trajectories, and the model will demonstrate the conditions of such deviations.

\section{Model of Deviation}

Many models and frameworks capture the interactive experience by people, for example as the M-Dimensions presented by Gonçalves et al [2]], which demonstrates the ten dimensions that guide and evaluate interactive installations, and a framework developed by [4] that captures factors that influence engagement. However, to our knowledge, there is a lack of a tool or a model that captures the deviation between the designer's design intentions and the participants' forms of engagement. They tend to come with different knowledge backgrounds and potentially with different purposes while looking at the same installation. We propose the 'deviation model' and suggest this model could help designers and curators bridge between the two trajectories.

We need to point out that the model proposed in this work will not focus on the comparison in terms of the degree of interactivity among the different installations, because some are meant to be less interactive by design [1] [3]. Instead, it will focus on the type and level of engagement by the participants and the extent the participants are engaged. In other words, the model focuses on the extent the participants explore and interact with the whole set of 
functionalities and experiences offered by a certain installation, no matter how simple or complex these interactions are. We further propose, as part of this model, a tool to visualize two trajectories: the intended experience by the designer and the actual experience with the installation in the real-world setting and the gap between. In the following, we first outline the process of engagement with interactive installations with its various stages. We then illustrate ways to visualize the two trajectories indicating the points of overlap and compatibility and the points deviation between a) designer's intention and b) actual experience by people who interact.

\subsection{The Interaction Process}

The fundamental part of this model is reflected as a linear exploration of the process of engagement. Drawing on Reeves et al who suggested that the interaction design process can be split into "manipulations" and "effects" [ $\underline{5}$, and Sharp et al who suggested that interactive design incorporates "user experience goals" and "usability goals" [6], both works demonstrated a consensus that engagement can be perceived as two major parts: the process of closely interacting with the installations in person and the point where certain outcomes are derived. These two major parts could be further expanded in which the process can be decomposed into five stages.

1- Lingering. As the very first stage, lingering usually contains approaching, by-standing and observing. The three can either happen at the same time or one after another.

2- Exploring. Exploring happens when the participant decides to figure out how the installation works and interact in person with it. Related behaviors include trying to press a button and reading instructions.

3- Direct Interaction. This means the participant is committed to the installation for the time being and deploys its functionalities. At this stage, the participant knows clearly what he/she is doing and ideally is generating the results as the designer prescribed.

4- Primary Outcome. This indicates that the first round of outcomes has been generated, which is sometimes implemented passively. For example, the participant feels certain emotions or acquiring certain skills are both the results that are derived directly from the experience itself.

5- Secondary Outcome. This stage can be seen as the "results of the first round of the outcome". After being affected directly by the installation and going through the fourth stage, the participant may react to the first round of interaction with personal actions, including taking photos, leaving comments, sharing the experience on social networks, etc.

According to Sharp et al, the first three stages can be perceived as "user experience goals" while the last two can be "usability goals". We extend Koeman's framework, which has four stages (discovery, understanding, interaction and sharing) [4]. We divide the "sharing" into two rounds of the outcome.

\subsection{The Visualization of Deviation}

The five stages can be illustrated as a linear process. The process so far reflects the anticipated interaction, an ideal condition intended by the designer. To measure and visualize the actual experience of a certain interaction trajectory, a line graph can be drawn with the linear five stages. The levels of deviations at the points are indicated with 'H' (Figure 1). The length of H represents the time he/she has spent on doing something that is out of the expectation of the designer, which can be described as "off-track".

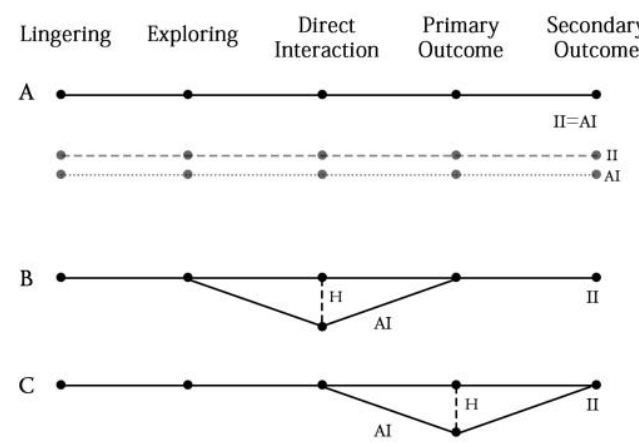

Figure 1: Line graphs indicating the deviation of engagement: II (upper trajectory): intended interactions by design, AI (lower trajectory): Actual interactions on-site, $\mathrm{H}$ : a gap that illustrates the deviation between II and AI. A(above): complete overlap at any stage between the intended interaction and the actual interaction; B(middle): the deviation between II and AI at the Direct Interaction stage; C(lower): the deviation between II and AI at the Primary Outcome stage 


\section{The Pilot Study: Flowing Mist}

To apply the model to a real-life installation, we carefully choose the installation of Flowing Mist at the Wonderlab of the Science Museum in London. To understand the intended interaction, we interviewed the curator and read the online and on-site instructions. The installation aims to demonstrate the features of mist by inviting people to blow the mist down the slope, while most participants, especially the children, interact with the slope in other ways. The installation was observed by one researcher for one hour, and the interaction stages were noted and captured using a camera. As can be derived from the graph, the participant stopped engaging with the installation at the third stage and thus did not generate the results as intended by the designer (ie learning about the features of the mist). This can be described as detachment, as the participant detached herself from the overall experience (Figure 2 ).
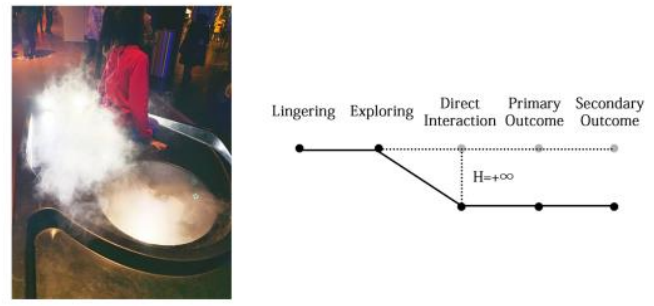

Figure 2 (left): An observation of one participant engaging in the Direct Interaction stage with the installation Flowing Mist. (right): the deviation diagram for this installation, She approached the installation but then started to slide herself down the slope instead of trying to play with or blowing the mist

However, although some users' actual experiences displayed a deviation at the Direct Interaction stage, they still generated the primary and secondary outcomes as expected, which avoided the situation of detachment between the intended interaction and the actual interaction. For example, instead of blowing the mist down the slope, some children tried to touch it, push it, smell it and watch it. Some were heard to say comments such as "it is wet!" and "it is light!", which showed that they did learn about the features of the mist without operating accurately as instructed.

\section{Conclusion}

In this paper we presented 'the deviation model' as a tool that can capture and visualize the difference between the experience trajectory with interactive installations 1) as anticipated by the designer and; 2) the actual experience of the participants on a variety of interaction stages. This difference can be referred to as the "deviation" between the two trajectories, and the model will demonstrate the conditions of such deviations.

This model is able to capture the pattern of participants' behavior when engaging with interactive installations. Participants may behave differently than initially intended prescribed by the designer. According to the level and condition of the engagement, the deviation can be visualized.

We presented a model that illustrates two interaction trajectories represented through engagement sequences, one as anticipated by the designer and the other one based on the observation of real-life interaction by one of the participants. We suggest that this model can offer new tools for designers and curators when designing for interactivity. At this stage, we show only one participant as a proof of concept, in the next stage of the research we intend to expand the framework and analyze a variety of installations, and compare the various stages, according to 1) the intention of the designers, and; 2) the actual interaction behavior with the installations by the people who engaged with the installation, audiences and context.

\section{REFERENCES}

$<$ bib id="bib1" $><$ number $>[1]</$ number $>$ Moritz Behrens, Ava Fatah gen. Schieck, and Duncan Brumby. 2015. Designing Media Architectural Interfaces for Interactions in Urban Spaces. In: Citizen's Right to the Digital City: Urban Interfaces, Activism, and Placemaking, 1st ed. Singapore: Springer Science+Business Media, pp.55-77.</bib $>$

$<$ bib id="bib2" $><$ number $>[2]<$ /number $>$ Harry Brignull and Yvonne Rogers. 2003. Enticing People to Interact with Large Public Displays in Public Spaces. In: INTERACT'03. Zurich: IOS Press, pp.17-24.</bib $>$

$<$ bib id="bib3" $><$ number $>[3]</$ number $>$ Lígia Gonçalves, Pedro Campos, and Margarida Sousa. 2012. M-dimensions: a Framework for Evaluating and Comparing Interactive Installations in Museums. In: Nordic Conference on HCI, pp.59-68.</bib>

$<$ bib id="bib4" $><$ number $>[4]</$ number $>$ Lisa Koeman. 2017. Urban Visualisation: the Role of Situated Technology Interventions in Facilitating Engagement with Local Topics. PhD Thesis. UCL.Conference Name:ACM Woodstock conference $</$ bib $>$

$<$ bib id="bib5" $><$ number $>[5]</$ number $>$ Stuart Reeves, Steve Benford, Claire O'Malley, and Mike Fraser. 2005. Designing the Spectator Experience. In: CHI'05. New York: ACM, pp.741-750Conference Name:ACM Woodstock conference</bib> 
$<$ bib id="bib6" $><$ number $>[6]</$ number $>$ Helen Sharp, Yvonne Rogers, and Jenny Preece. 2007. Interaction Design: Beyond Human-Computer Interaction, 2nd Edition / Sharp, Helen. 1st ed. $<$ /bib $>$

$<$ bib id="bib7"><number $>[7]</$ number $>$ Martin Tomitsch, Christopher Ackad, Oliver Dawson, Luke Hespanhol, and Judy Kay. 2014. Who Cares about the Content? An Analysis of Playful Behaviour at a Public Display. In: PerDis '14. New York: ACM, pp.160-165.Conference Name:ACM Woodstock conference</bib $>$ 